\title{
Localization of PRAMEY Protein in Bovine Testis by Immunoelectron Microscopy
}

Gang Ning and Wan-Sheng Liu

Pennsylvania State University, University Park, Pennsylvania, United States

PRAME (preferentially expressed antigen in melanoma) is a cancer/testis antigen (CTA) that is predominantly expressed in normal germinal tissues and a variety of tumors. Like other CTAs, PRAME is a multicopy gene family representing one of the most amplified gene families in eutherian mammals. Members of the PRAME gene family encode leucine-rich repeat (LRR) proteins that fold into a horseshoe shape and provide a versatile structural framework for the formation of protein-protein interactions. PRAME has been extensively studied in cancer biology and is believed to play a regulatory role in cancer cells [1]. Nevertheless, its germinal tissue-specific expression, the function of PRAME during spermatogenesis remains unknown. In bovid lineage, PRAME was transposed to the Y chromosome through an 'autosome-to-Y' transposition mechanism during evolution. The Y-linked gene, or PRAMEY, was amplified on the $\mathrm{Y}$ chromosome with a copy number variation ranging from 2 to 31 among cattle breeds. The CNVs of PRAMEY were found recently associated with testis size and male fertility.

In order to study the functional role of PRAMEY during spermatogenesis, we have generated and validated an antibody for the bovine PRAMEY. Our previous western blot analysis at different developmental stages $(20 \mathrm{~d}, 3 \mathrm{~m}, 8 \mathrm{~m}$ and $2 \mathrm{y}$ ) of the bovine testes indicated that PRAMEY has four different isoforms $(58,30,26$, and $13 \mathrm{kDa})$. Of which the intact protein $58 \mathrm{kDa}$ was expressed in all age of testes, while the $30 \mathrm{kDa}$ was first expressed at the age of puberty ( 8 months) as well as in adult testes. The 26 and $13 \mathrm{kDa}$ were expressed in epidydimal spermatozoa [2]. Immunofluorescence microscopy further revealed that PRAMEY was expressed in the acrosome of spermatids and mature spermatozoa [3]. In the present study, we used immunoelectron microscopy to localize PRAMEY protein in bovine testis.

The bovine testis tissues were obtained from adult animals from a local slaughterhouse. The tissue blocks were fixed with $0.1 \%$ glutaraldehyde and $4 \%$ paraformaldehyde, and then cryo-protected with $2.3 \mathrm{M}$ sucrose and frozen in liquid propane cooled in liquid nitrogen. The tissue blocks were processed in a Leica automatic freeze-substitution system and embedded in Lowicryl HM20 at low temperature. Immunolabeling for electron microscopy were performed with thin sections on EM grids. The results showed the PRAMEY was specifically localized in spermatids: the immunolabeling was clearly found in some amorphous, non-membrane bound structures in the cytoplasm of round spermatids, and then was highly concentrated in acrosomal granules and matrix in elongated spermatids (Figures). These results suggest PRAMEY is expressed and plays a role in spermiogenesis, i.e., the transformation of spherical, haploid spermatids into elongated, highly condensed and mature spermatozoa [4]. 

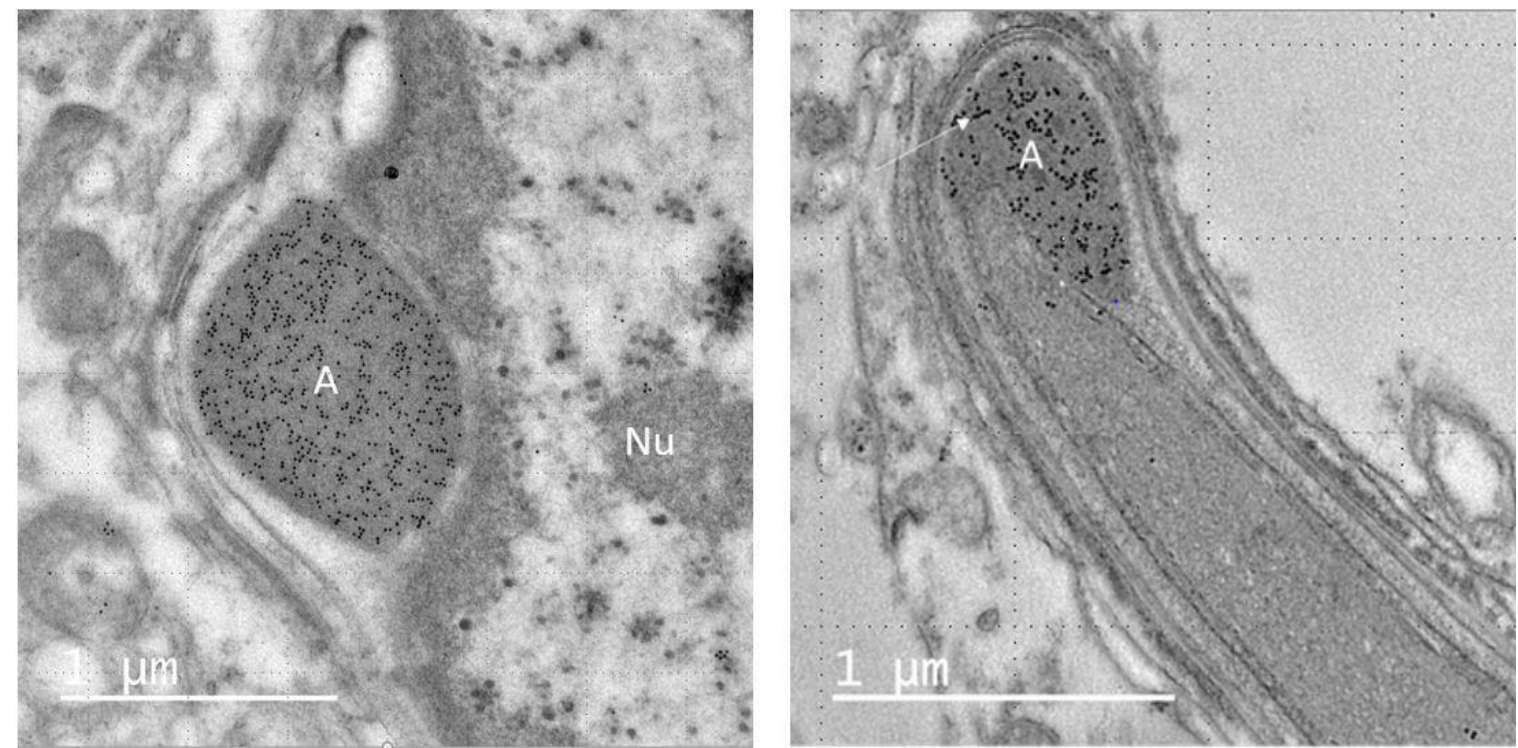

Figure 1. TEM micrographs show PRAMEY are specifically localized in acrosomal granules (A) of round spermatid (left) and elongated spermatid (right) by immunogold particles. $\mathrm{Nu}$, nucleus.

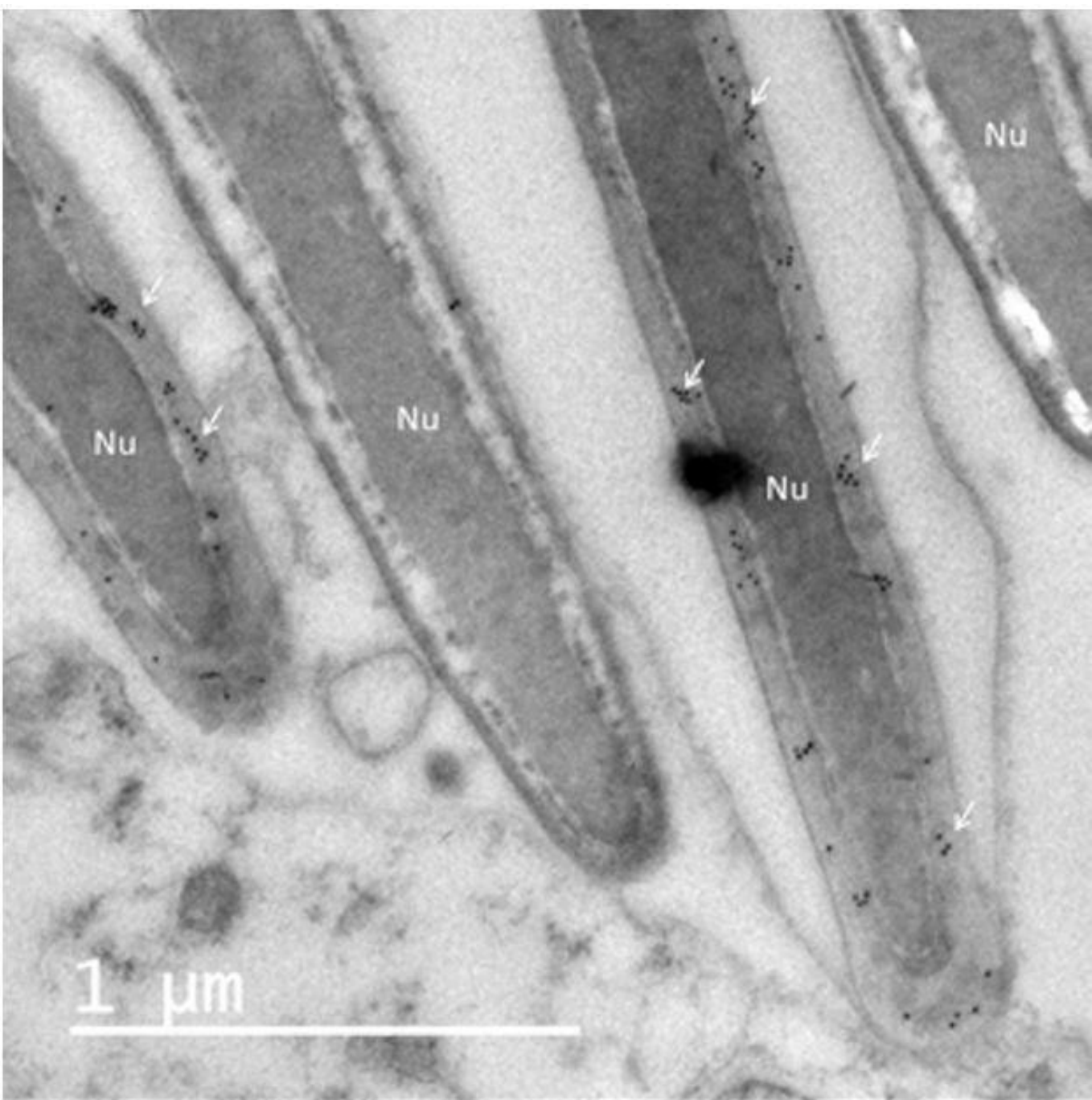


Figure 2. Four elongated spermatids are seen in the micrograph. Two of them are labeled with gold particles (arrows) in acrosomal matrix, while the other two are not labeled because their acrosomes are not visible.

\section{References}

[1] Wadelin F et al. Molecular Cancer (2010) 9:226,

[2] Liu et al. Reproduction (2017) 153 847-863.

[3] Mistry BY et al. PLoS One (2013) 8 (4):e60611.

[4] The authors acknowledge funding from USDA-NIFA (\#2010-65205-20362) for WSL, and the Huck Institutes of the Life Sciences at Penn State and the Pennsylvania Department of Health using Tobacco CURE funds for WSL and GN. Special thanks to Yaqi Zhao for her assistance in processing of the samples. 